\title{
Improving the Processing Power Efficiency of Minimum Selection GSC with Adaptive Modulation and Post-Combining Power Control*
}

\author{
Zied Bouida, Mohamed-Slim Alouini, Khalid A. Qaraqe \\ Dept. of Electrical Eng. \\ Texas A\&M University at Qatar \\ Education City, Doha, Qatar \\ Email: \{zied.bouida, alouini, khalid.qaraqe\}@qatar.tamu.edu
}

\begin{abstract}
Many joint adaptive modulation and diversity combining (JAMDC) schemes have been recently proposed to improve the performance and efficiency of wireless communication systems. Considering the problem of finding low-complexity, bandwidth-efficient, and processing-power efficient transmission schemes for a downlink scenario and capitalizing on one of these recently proposed JAMDC schemes, we propose and analyze in this paper a JAMDC scheme using post combining power control and finger deactivation. More specifically, the modulation constellation size and the number of combined diversity paths are jointly determined to achieve the highest spectral efficiency with the lowest possible combining complexity, given the fading channel conditions and the required error rate performance. Selected numerical examples show that the newly proposed scheme considerably reduces the processing power consumption with a slight increase in the radiated power while maintaining high average spectral efficiency and compliance with bit error rate constraints.
\end{abstract}

Index Terms-Diversity Techniques, Adaptive Modulation, Power Control, Finger Deactivation, and Performance Analysis.

\section{INTRODUCTION}

Future wireless communication systems which will provide multimedia services to the power/size limited mobile terminals are characterized by limited bandwidth and power resources. These systems should be able to support high spectral efficiency with good link reliability. This need for higher bandwidth efficiency motivates further optimization of the use of wireless resources. Due to user mobility and highly time-variant propagation environments, resource management in wireless communications becomes a difficult task which implies adaptive solutions. Key adaptive solutions are those of adaptive modulation [1], [2], power control [3], [4], and adaptive combining [5]-[8].

Based on multiple thresholds, adaptive modulation can achieve high spectral efficiency over wireless channels. The key idea of adaptive modulation is to adapt the modulation parameters, such as constellation size, to fading channel conditions while respecting the bit error rate (BER) requirements. Diversity combining, on the other hand, improves the reliability of wireless fading channels by adapting the combiner structure to fading channel conditions. Adaptive power control

* This work was supported in part by the Qatar Foundation for Education, Science, and Community Development, Qatar, and in part by Qatar Telecom (Qtel), Qatar. schemes, unlike schemes using a constant-power variablerate setup, adapt the transmitted power to fading channels conditions while fulfilling the BER constraint. These schemes reduce the radiated power, and thus the potential interference to other systems/users which implies a significant network capacity improvements.

These adaptive solutions have been originally studied separately. Recently, joint adaptive solutions have been proposed and studied. For instance, while joint adaptive modulation and combining schemes were introduced in [9], [10], joint adaptive combining and power control were studied for constant-rate transmission in [11]-[13]. In addition, in [14] and for the purpose of interference reduction, Gjendemsjø et. al. extended the schemes discussed in [9]-[13] by looking at joint adaptive modulation, diversity combining, and power control. Capitalizing on this most recent work, and in order to have more processing power efficiency, we propose in this paper a modified bandwidth-efficient scheme using finger deactivation (BES-FD). We analyze this newly proposed scheme in term of average spectral efficiency (ASE) (in bits/s/Hz), average BER, diversity combining complexity, and transmit power gain and compare its performance to that of the bandwidthefficient scheme (BES JAMDC) proposed in [14]. Selected numerical examples show that the BES-FD scheme reduces the processing power consumption of the BES scheme with a slight increase in the radiated power, while keeping a high ASE and compliance with BER constraints.

The remainder of the paper is organized as follows. Section II presents first the system and channel models then gives the details behind the adaptive transmission system, the mode of operation of the proposed scheme, and power control. Next, the performance analysis of the proposed scheme is given in section III. Section IV offers some selected numerical examples illustrating the performance of BES-FD and comparing it to that of BES JAMDC. Finally, section V concludes the paper.

\section{MODELS AND MODE OF OPERATION}

\section{A. System and Channel Model}

We consider a generic diversity system with $L$ available diversity paths and we assume that the proposed BES-FD scheme has a reliable feedback path between the receiver and the transmitter and are implemented in a discrete-time fashion. More specifically, short guard periods are periodically inserted into the transmitted signal. During these guard 
periods, the receiver performs a series of operation, including (i) path estimation, (ii) decision on a diversity structure and signal constellation, and (iii) selection of the power to be used for transmission. Once the suitable paths for combining and constellation size are selected and once the appropriate transmitted power is fixed, the combiner and the demodulator (at the receiver end) and the high power amplifier (HPA) and the modulator (at the transmitter) are configured accordingly and these settings are used throughout the subsequent data burst. Under the assumption of frequency flat fading, we use a block-fading model assuming that different diversity paths experience roughly the same fading conditions (or equivalently the same SNR) during the data burst and its preceding guard period. For our study, we assume that the received signal on each diversity branch experiences independent identically distributed (i.i.d.) Rayleigh fading.

\section{B. Adaptive Transmission System}

We consider the constant-power variable-rate $M$-ary QAM [1] as an adaptive modulation system for our proposed adaptive transceiver. With this adaptive modulator, the SNR range is divided into $N+1$ fading regions and the constellation size $M=2^{n}$ (where $n$ is the number of bits per symbol) is assigned to the $n$th region $(n=0,1, \ldots N)$. The selection of a constellation size is based on the fading channel state. Specifically, we partition the range of the SNR after diversity combining into $N+1$ regions, which are defined by the switching thresholds $\left\{\gamma_{T_{n}}\right\}_{n=1}^{N}$, and transmit using constellation $n$ if the combined SNR is in the interval $\left[\gamma_{T_{n}}, \gamma_{T_{n+1}}\right)$.

The BER of $2^{n}$-QAM constellations with SNR of $\gamma$ is given in [1] by

$$
\operatorname{BER}_{n}(\gamma)=\frac{1}{5} \exp \left(\frac{-3 \gamma}{2\left(2^{n}-1\right)}\right)
$$

Given a target instantaneous $\mathrm{BER}$ equal to $\mathrm{BER}_{0}$, the region boundaries (or adaptive modulator switching thresholds) $\gamma_{T}$ for $n=0,1, \ldots N$ are given in this case by

$$
\gamma_{T_{n}}=-\frac{2}{3} \ln \left(\mathrm{BER}_{0}\right)\left(2^{n}-1\right) ; n=0,1, \ldots N \text {. }
$$

\section{Mode of Operation}

The mode of operation of the BES-FD is summarized in a flowchart given in Fig. 1. In the beginning of each data burst, the base station transmits a training sequence using the highest power level. After estimating and ranking the $L$ available paths, the combiner in the mobile's side tries to increase the output SNR above the threshold for the highest constellation size by performing the minimum selection generalized selection combining (MS-GSC) scheme, proposed in [5] and further studied and analyzed in [6]-[8], with $\gamma_{T_{N}}$ as output threshold. Whenever the combined SNR is larger than $\gamma_{T N}$, the receiver selects the highest constellation size $(N)$ and asks the transmitter to use the lowest possible power level such that the highest modulation mode ( $2^{N}$-QAM) is still usable. If the combined SNR of all available branches is still below $\gamma_{T N}$, the mobile determines the highest feasible constellation size by comparing the combined SNR to different switching thresholds
$\left\{\gamma_{T_{n}}\right\}_{n=1}^{N-1}$. The modulation mode $n$ is selected by the mobile if the combined SNR is smaller than $\gamma_{T_{n+1}}$ but greater than $\gamma_{T_{n}}$. The mobile will then start the finger deactivation process by selecting the minimum number of paths that are needed such that the output SNR is still greater than $\gamma_{T_{n}}$ (i.e turning off the weakest branches while conserving the same modulation mode). The deactivation process is continued until turning off a further diversity path leads to an output SNR below $\gamma_{T_{n}}$. After this, the base station reduces its power level such that the selected constellation is still usable. If even the lowest constellation size is not feasible, data is buffered, and there is no transmission for the next time interval.

\section{Power Control}

We use for the BES-FD scheme the same power adaptation variants used for the BES JAMDC with post combining power control of [14]. It means that we analyze both continuous and discrete power adaptation.

The post-adaptation power control is defined by $\Gamma^{\prime}=\frac{\Gamma}{\beta}$, where $\Gamma$ is the combined SNR with BES-FD before power control and $\beta$ is the power control parameter. While for continuous adaptation $\beta \in[1, \infty)$, for the discrete power adaptation there are $M$ power parameters: $\left\{\beta_{1}=1<\beta_{2}<\right.$ $\left.\ldots<\beta_{M}\right\}$. If the modulation mode $n$ is selected then the SNR after continuous power control is reduced to $\gamma_{T}$. For the discrete adaptation the SNR will be reduced by $\beta_{i}$, where $\beta_{M}$ must verify the constraint given in [14] by

$$
\beta_{M} \leq \min _{1 \leq n \leq N} \frac{\gamma_{T_{n+1}}}{\gamma_{T_{n}}}
$$

\section{PERFormance ANALYSis}

\section{A. Transmit Power Gains}

1) Continuous Power Control: Using [14, Eq. (6)] and the mode of operation of BES-FD, the average $\mathrm{dB}$ transmit power gain can be shown to be given by

$$
\begin{aligned}
\bar{G}_{\mathrm{dB}}= & \sum_{n=1}^{N} \int_{\gamma_{T_{n}}}^{\gamma_{T_{n+1}}} 10 \log _{10}\left(\frac{\gamma_{c}}{\gamma_{T_{n}}}\right) f_{\Gamma}(x) d \gamma_{c} \\
= & \sum_{n=1}^{N} \int_{\gamma_{T_{n}}}^{\gamma_{T_{n+1}}} 10 \log _{10}\left(\gamma_{c}\right) f_{\Gamma}(x) d \gamma_{c} \\
& -\sum_{n=1}^{N} 10 \log _{10}\left(\frac{\gamma_{c}}{\gamma_{T_{n}}}\right)\left(F_{\Gamma}\left(\gamma_{T_{n+1}}\right)-F_{\Gamma}\left(\gamma_{T_{n}}\right)\right)
\end{aligned}
$$

where $F_{\Gamma}($.$) and f_{\Gamma}($.$) are the cumulative distribution function$ (CDF) and the probability density function (PDF) of the combined SNR with BES-FD, respectively, and are the same as those of the bandwidth efficient and power greedy scheme proposed in [10].

2) Discrete Power Control: The average $\mathrm{dB}$ transmit power gain for discrete adaptation is derived from [14, Eq. (8)] and 
is given by

$$
\begin{aligned}
\bar{G}_{\mathrm{dB}}= & \sum_{n=1}^{N} \int_{\gamma_{T n}}^{\gamma_{T n+1}} 10 \log _{10}(\beta) f_{\Gamma}(x) d \gamma_{c} \\
= & \sum_{n=1}^{N} \sum_{m=1}^{M} 10 \log _{10}\left(\beta_{m}\right) \\
& \times\left(F_{\Gamma}\left(\gamma_{T_{n}} \beta_{m+1}\right)-F_{\Gamma}\left(\gamma_{T_{n}} \beta_{m}\right)\right),
\end{aligned}
$$

where $\gamma_{T_{n}} \beta_{M+1}=\gamma_{T_{n+1}}$.

\section{B. Spectral and Processing Power Efficiency}

The BES-FD has the same spectral efficiency as the BES JAMDC scheme and the average spectral efficiency is given by

$$
\eta=N-\sum_{n=1}^{N} F_{\gamma_{c}}^{M S C\left(\gamma_{T_{N}}\right)}\left(\gamma_{T_{n}}\right)
$$

The average number of combined branches is given by

$$
\overline{N_{c}}=\sum_{l=1}^{L} \sum_{n=1}^{N} l P_{l, n},
$$

where $P_{l, n}$ is given in [10, Eqs. (25), (26), (27)].

\section{Bit Error Rate}

The average BER of the BES-FD scheme can be calculated as $[1$, Eq. (35)]

$$
\overline{\mathrm{BER}}=\frac{1}{\eta} \sum_{n=1}^{N} n \overline{\mathrm{BER}}_{n},
$$

where $\overline{\mathrm{BER}}_{n}$ is the average BER for constellation size $n$ as

$$
\overline{\mathrm{BER}}_{n}=\int_{\gamma_{T_{n}}}^{\gamma_{T n+1}} \mathrm{BER}_{n}\left(\gamma^{\prime}\right) f_{\Gamma^{\prime}}\left(\gamma^{\prime}\right) d \gamma^{\prime},
$$

where $f_{\Gamma^{\prime}}($.$) is the PDF of the combined SNR with BES-FD$ after power control and which is obtained for continuous and discrete power control by [14] Eqs. (12) and (17), respectively, and by replacing the statistics of the combined SNR with BES JAMDC by the statistics of the combined SNR with BES-FD. Using Eq. (9) in Eq. (8) we can write

$$
\overline{\mathrm{BER}}=\frac{\sum_{n=1}^{N} n \int_{\gamma_{T_{n}}}^{\gamma_{T_{n+1}}} \mathrm{BER}_{n}\left(\gamma^{\prime}\right) f_{\Gamma^{\prime}}\left(\gamma^{\prime}\right) d \gamma^{\prime}}{\eta}
$$

\section{NumericAl EXAMPLES}

For these examples, we set the number of available diversity branches $L=3$, the number of signal constellations $N=4$, and the bit error rate constraint as $\mathrm{BER}_{0}=10^{-3}$

While we can see from Fig. 2 that the BES-FD scheme conserves the same spectral efficiency as the BES JAMDC scheme, Fig. 3 shows that the proposed scheme considerably reduces the average number of combined branches (i.e offers some processing power savings) in the $0-20 \mathrm{~dB}$ SNR range.
These results are explained by the fact that the finger deactivation process turns off the weakest branches while conserving the same constellation size. For an average SNR above 20 $\mathrm{dB}$, we can see that for both schemes one diversity path is enough to utilize the highest constellation size (i.e 16-QAM modulation).

In Fig. 4, we depict the average transmit power gain versus the average SNR per branch for the BES-FD scheme for both, continuous and discrete, adaptations. The shape of the graphs in this figure is the same as in [14, Fig. (6)], but with lower values for the average transmit gain. This shows that the BES-FD scheme has lower average transmit power gain, or equivalently higher average radiated power, than the BES JAMDC scheme.

According to the constraint given in (3), the maximum reduction for discrete level transmit power control is limited by the length of the shortest interval. This explains why the average transmit power gain saturates in different values depending on the used power control step size.

In Fig. 5, we show the bit error performance of the proposed scheme and compare it to this of the BES JAMDC scheme. For continuous power control adaptation the proposed BESFD has the same BER performance as the BES JAMDC scheme. For this case, the BER is constant and is equal to $\mathrm{BER}_{0}$, since the combined SNR after finger deactivation and continuous power control will be set to the switching threshold corresponding to the used constellation. For discrete power control adaptation we show that the BES-FD scheme has poorer error performance than the BES JAMDC scheme. For reference, we also compare the BER performances of the BES-FD and the BES JAMDC schemes using constant full power.

\section{CONCLUSION}

We have proposed in this paper a variant of the existent BES JAMDC scheme by the introduction of the finger deactivation process. Selected numerical examples show that the proposed scheme conserves the same spectral efficiency as the BES JAMDC scheme. On the other hand, the BES-FD scheme offers better power efficiency at the cost of a slightly higher radiated power and slightly poorer BER. In conclusion, while $B E S$ JAMDC gives higher average transmit power gains BESFD offers considerable processing power savings.

\section{REFERENCES}

[1] M.-S. Alouini and A. J. Goldsmith, "Adaptive modulation over Nakagami fading channels," Kluwer J. Wireless Communications, vol. 13 pp. 119-143, May 2000.

[2] K. J. Hole, H. Holm, and G. E. Øien, "Adaptive multidimensional coded modulation over flat fading channels," IEEE J. Select. Areas Commun. vol. 18 , no. 7 , pp. $1153-1158$, July 2000 .

[3] A. Gjendemsjø, G. E. Øien, and H. Holm, "Optimal power control for discrete-rate link adaptation schemes with capacity-approaching coding," in Proc. IEEE Global Telecommunications Conference (GLOBECOM'05), St. Louis, MO, Nov.-Dec. 2005, pp. 3498-3502.

[4] A. Gjendemsjø, G. E. Øien, and P. Orten, "Optimal discrete-level power control for adaptive coded modulation schemes with capacity approaching component codes," in Proc. IEEE International Conference on Communications (ICC'06), Istanbul, Turkey, March 2007.

[5] S. W. Kim, D. S. Ha, and J. H. Reed, "Minimum selection GSC and adaptive low-power RAKE combining scheme," in Proc. IEEE Int. Symp. on Circuit and Systems (ISCAS'03), Bangkok, Thailand, May 2003. 
[6] P. Gupta, N. Bansal, and R. K. Mallik, "Analysis of minimum selection H-S/MRC in Rayleigh fading," IEEE Trans. Commun., vol. 53, pp. 780784, May 2005.

[7] R. K. Mallik, P. Gupta, and Q. T. Zhang, "Minimum selection GSC in independent Rayleigh fading," IEEE Trans. Veh. Technol., vol. 54, no. 3, pp. 1013-1021, May 2005.

[8] H.-C. Yang, "New results on ordered statitics and analysis of minimum selection generalized selection combining (GSC)," IEEE Trans. Wireless Commun., vol. 5, no. 7, pp. 1876-1885, July 2006.

[9] N. Belhaj, N. Hamdi, M.-S. Alouini, and A. Bouallegue, "Low-power minimum estimation and combining with adaptive modulation," in Proc. IEEE Int. Symp. on Signal Processing and its Applications, Sydney, Australia, August 2005, pp. 687- 690.

[10] H.-C. Yang, N. Belhaj, and M.-S. Alouini, "Performance analysis of joint adaptive modulation and diversity combining over fading channels," in Proc. International Wireless Communications and Mobile Computing Conference, Vancouver, BC, Canada, July 2006.

[11] N. Belhaj, M.-S. Alouini, and K. Qaraqe, "Minimum selection GSC with down-link power control," in Proc. IEEE Vehicular Tech. Conf. (VTC'06-Spring), Melbourne, Australia, May 2006.

[12] S. S. Nam, M.-S. Alouini, and K. Qaraqe, "Diversity combining with up-link power control," in Proc. IEEE International Symposium on Personal, Indoor and Mobile Radio Communications, Helsinki, Finland, September 2006.

[13] Z. Bouida, M.-S. Alouini, and K. Qaraqe, "Joint minimum selection GSC and down-link power control," in Signal Processing for Wireless Communications Workshop, London, England, June 2007.

[14] A. Gjendemsjø, H. C. Yang, G. E. Øien, and M.-S. Alouini, "Minimum selection GSC with adaptive modulation and post-combining power control," in Proc. of IEEE Wireless Communications and Networking Conference (WCNC'2007), Hong Kong, China, March 2007.

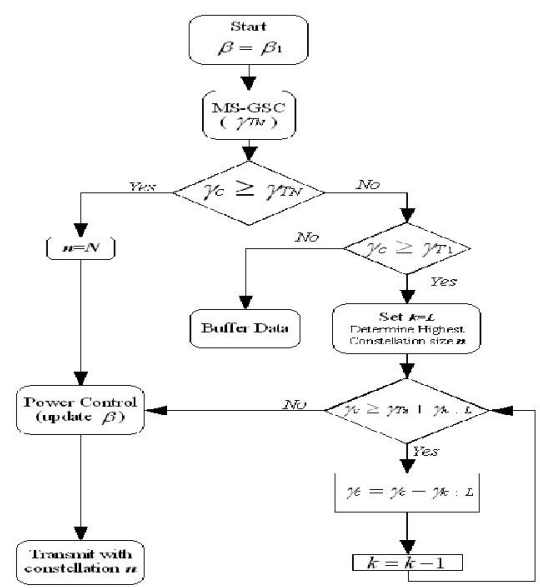

Fig. 1. Mode of operation of the BES-FD scheme.

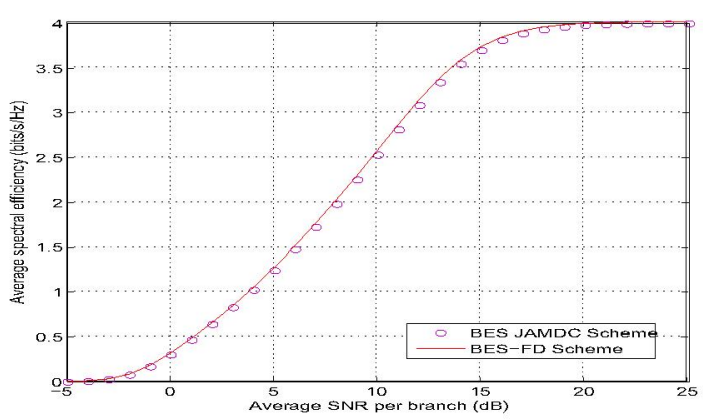

Fig. 2. Average spectral efficiency versus the average SNR per branch, $\bar{\gamma}$, with BES JAMDC and BES-FD when $L=3$ and $N=4$.

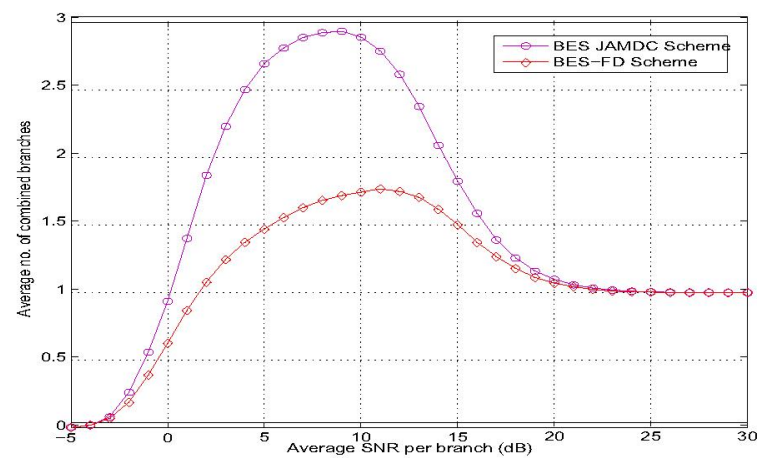

Fig. 3. Average number of combined paths versus the average SNR per branch, $\bar{\gamma}$, with BES JAMDC and BES-FD when $L=3$ and $N=4$.

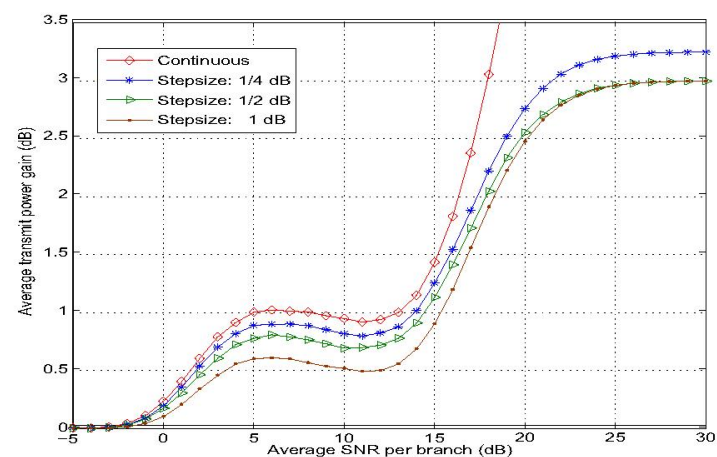

Fig. 4. Average transmit power for the BES-FD scheme versus the average SNR per branch, $\bar{\gamma}$, when $L=3$ and $N=4$.

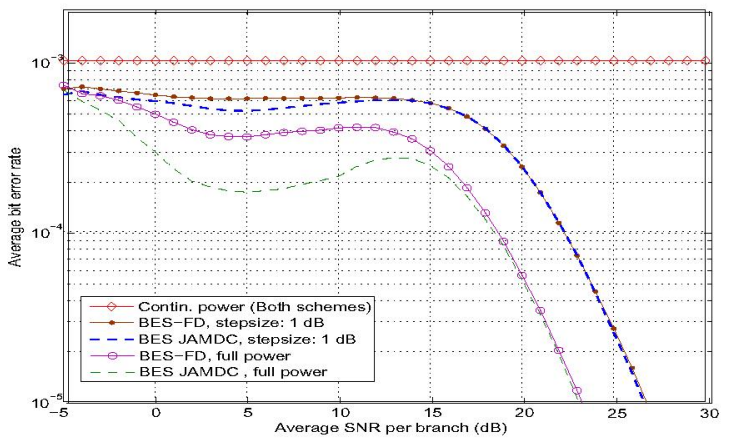

Fig. 5. Average bit error rate versus the average SNR per branch, $\bar{\gamma}$, with BES JAMDC and BES-FD when $L=3, N=4$, and a BER constraint $\mathrm{BER}_{0}=10^{-3}$ 